\title{
Why are we here? A study of patient actions prior to emergency hospital admission
}

\author{
J R Benger, ${ }^{1,2} \mathrm{~V}$ Jones $^{3}$
}

\begin{abstract}
- Additional Appendix is published online only at http:// emj.bmj.com/content/vol25/ issue7

${ }^{1}$ United Bristol Healthcare Trust, Bristol, UK: ${ }^{2}$ University of the West of England, Bristol, UK;

${ }^{3}$ Academic Department of Emergency Care, United Bristol Healthcare Trust, Bristol, UK
\end{abstract}

Correspondence to: Dr J R Benger, Emergency Department, Bristol Royal Infirmary, Bristol BS2 8HW, UK; Jonathan.Benger@ubht.nhs.uk

Accepted 9 December 2007

\begin{abstract}
Introduction: Emergency department (ED) attendances and subsequent hospital admissions are rising in the United Kingdom. The reasons for this are unclear but may relate to recent changes in primary care and public perception. The actions taken by patients or their relatives before emergency hospital admission, the reasons for these actions and their outcome were determined. Methods: Adult patients admitted to an inner city teaching hospital with a medical or surgical illness were interviewed using a semistructured questionnaire. Data were collected and analyzed regarding the actions taken before arrival at hospital, the reasons for taking these actions, their outcome and future intentions. 200 patients were interviewed.
\end{abstract}

Results: Direct attendance at the ED was more common when help was sought by bystanders or persons known only slightly to the patient $(p=0.03)$. 57 patients $(28.5 \%)$ attended the ED directly, 45 of whom dialled 999 for an emergency ambulance. Most patients who attended the ED directly did so as a result of the perceived severity or urgency of their condition and there was incomplete awareness of the out-of-hours GP service.

Conclusion: The majority of adult patients who are admitted to hospital with an acute illness seek professional help from primary care in the first instance. Those who attend the ED generally perceive their problem as more urgent or severe, or have an ambulance called on their behalf. The shift towards ED care appears partly driven by changes in general practice and unfamiliarity with the new arrangements for out-of-hours primary care provision.

In the United Kingdom patient attendances at hospital emergency departments (ED) continue to rise year on year. In England new ED attendances increased from 15.3 million in the year 2003/4 to 16.7 million in the year 2004/5 (an increase of 9\%). ${ }^{1}$ Similarly, the number of patients admitted to hospital from major English ED increased from 640840 in the final quarter of the year 2003/4 to 724814 in the final quarter of 2004/5 (an increase of $13 \%)^{2}$ This increase has occurred despite numerous initiatives that have been introduced in an attempt to prevent emergency admission to hospital. ${ }^{3}$

The reason for steadily increasing ED attendances and hospital admissions is often debated but poorly informed by evidence. Changes such as an ageing population, shifts in patient expectations and reconfiguration of primary care services have all been cited. ${ }^{4}$ Within primary care, it has been suggested that the introduction of the "24/48" access target, by which patients should see a primary healthcare professional within $24 \mathrm{~h}$ and a general practitioner (GP) within $48 \mathrm{~h}$ have conversely led to service reconfigurations that may increase ED attendance..$^{5}$ Similarly, changes in primary care mean that only a minority of GPs now undertake out-of-hours work, with Primary Care Trusts commissioning alternative means of provision that may be viewed as less accessible or familiar to the general public

In our local health community the view had arisen that patients were bypassing primary care and attending the ED "inappropriately". It was therefore proposed that if patients with urgent healthcare needs accessed primary care in the first instance this would reduce hospital attendance and admission. Indeed, this view continues to be reflected in UK policy documents. ${ }^{6}$ Our research was therefore undertaken to determine the route by which patients with acute illness are admitted to hospital. In particular, we sought to examine the reasons for the various actions taken and the outcome of these actions, to examine the extent to which patient behaviour and referral pathways may be contributing to increased ED attendances and hospital admissions.

\section{METHODS}

This study was carried out over 4 weeks during October and November 2005 at the Bristol Royal Infirmary, a city-centre teaching hospital in southwest England. Adult patients being admitted to hospital after attendance at the ED, and those admitted directly by their GP to a medical or surgical bed, were eligible for inclusion. Patients were recruited consecutively during representative time periods between 08:00 hours and midnight on all 7 days of the week. After informed, written consent had been obtained, each patient or accompanying relative was administered a semistructured questionnaire (reproduced in Appendix 1 , available online only) to determine the actions taken before arrival at hospital, the reasons for doing so, the outcome of these actions and future intentions should the same situation arise again.

Exclusion criteria were as follows: patients in triage category 1 (these are the most severely ill patients and were considered too ill to participate); patients under 16 years of age; patients admitted as a result of injury; patients admitted as a result of mental illness; patients who declined to take part.

Data were analyzed using descriptive statistics, with additional Spearman rho, Mann-Whitney, Kruskal-Wallis and $\chi^{2}$ testing according to the data type. Prespecified analyses included a comparison of patients attending the ED directly with those who initially contacted their GP's surgery, 


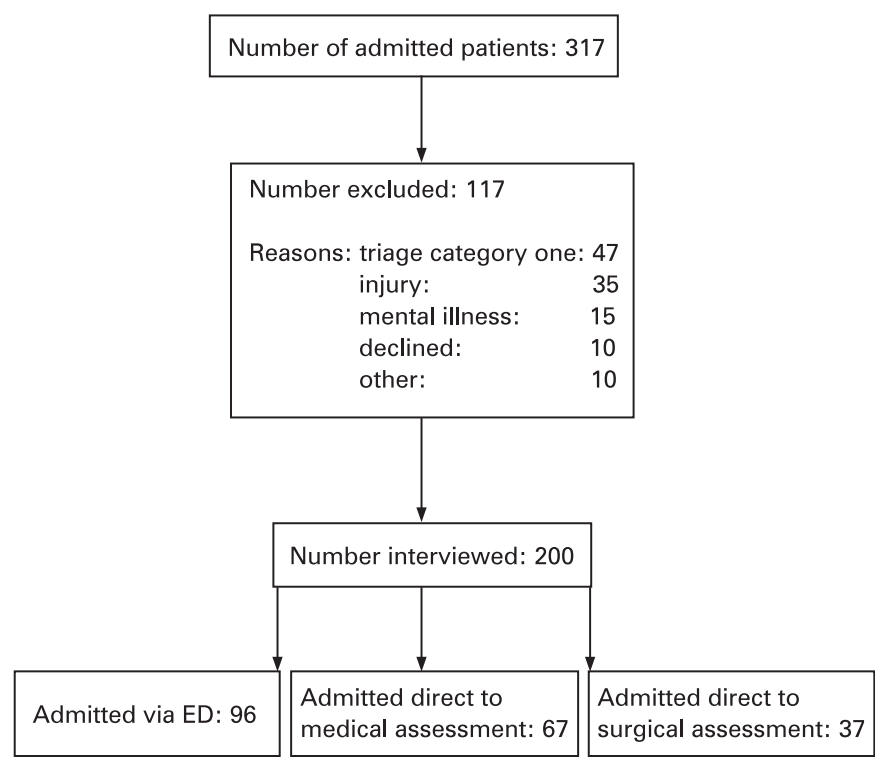

Figure 1 Flow of study patients. $E D$, emergency department.

either in or out of hours. Free text responses were transcribed and analyzed to identify the most common themes.

\section{RESULTS}

During the 4-week study period 200 patients were successfully recruited. The flow of patients is shown in fig 1 .

The mean patient age was 58 years and the median was 61 years with a range of 16-91 years; 104 patients (52\%) were female; 108 patients (54\%) sought help themselves, whereas in 70 cases $(35 \%)$ friends or family sought professional help on behalf of the patient. In the remaining 22 patients (11\%) help was sought by bystanders or other individuals not well known to the ill person. The person seeking help had a statistically significant effect on the type of help sought, with patients themselves choosing to attend the ED $21 \%$ of the time, rising to $34 \%$ for relatives and friends and $52 \%$ for bystanders or less well-known people ( $p=0.03$ on $\chi^{2}$ testing).

The action taken by the person seeking professional help and the outcome of this action is shown in fig 2. A total of 102 patients (51\%) contacted their GP's surgery in normal working hours and 12 (6\%) out of hours; 57 patients (28.5\%) went directly to the $\mathrm{ED}$, whereas $29(14.5 \%)$ took another or combination of actions, including contacting NHS Direct or attending a walk-in centre. There was a tendency for older patients to seek help from their GP in normal working hours, whereas younger people contacted the out-of-hours primary care service or took other actions. This did not, however, achieve statistical significance $(p=0.11$ on Kruskal-Wallis testing). Similarly, direct attendance at the ED was more likely when help was sought out of hours compared with in hours, but this also did not achieve statistical significance ( $p=0.19$ on $\chi^{2}$ testing).

The most common reasons why patients chose to dial 999 or attend the ED directly ( $\mathrm{n}=57$ ) are shown in table 1 , and the most common reasons why patients chose to contact their GP in normal office hours $(\mathrm{n}=102)$ are shown in table 2 .

In the 12 patients contacting out-of-hours primary care the most common reasons were: a perceived need to seek medical advice regarding a problem that was not viewed as particularly urgent or severe (three patients); an inability to attend the GP
Table 1 Top five reasons why patients chose to dial 999 or attend the ED directly $(\mathrm{n}=57)$

\begin{tabular}{ll}
\hline Reason & No (\%) \\
\hline Perceived severity or urgency of their condition & $29(51)$ \\
Previous experience & $7(12)$ \\
Ease and convenience & $4(7)$ \\
Housebound & $4(7)$ \\
Primary care services are not available out of hours & $4(7)$ \\
\hline ED, emergency department. &
\end{tabular}

due to lack of mobility (three patients) and a view that calling the GP is the correct first step in accessing healthcare (two patients).

For patients who did not contact their GP first $(n=72)$ the most common reasons are shown in table 3.

Willingness to take the same action again in those attending the ED directly, or contacting their GP in hours, was generally high, with $75 \%$ and $73 \%$ saying that they would do so again. This contrasted with those contacting out-of-hours primary care, or taking another action, who stated that they would take the same action again in $36 \%$ and $38 \%$ of cases.

\section{DISCUSSION}

This study describes the route of access for adult patients admitted to hospital with acute illness. The majority of admitted patients (57\%) contacted their GP's surgery first, with a further $28.5 \%$ attending the ED directly, the majority of these doing so by 999 ambulance. The strongest factor influencing direct ED attendance was the individual who summoned help: bystanders encountering a person with sudden illness in the community are likely to dial 999 , whereas patients and their relatives prefer to access primary care.

A significant proportion of patients interviewed had contacted their GP's surgery in or out of hours and had either been advised to attend the ED immediately, or were admitted directly to hospital without being seen by a GP. In cases in which the need for ED attendance or hospital admission is clear this could be considered an efficient use of GP time and is sometimes of benefit to the patient. ${ }^{7}$ However, it also gives an impression that direct ED attendance is the correct course of action when unscheduled care needs arise. This is supported by the opinion of some patients that contacting their GP would lead to long delays or inevitable ED referral. In addition, the intended future actions of patients indicated a general trend towards increasing ED use.

The out-of-hours GP service proved to be a relatively minor source of emergency admissions, although data were not collected between midnight and 08:00 hours. It is impossible to tell whether this reflects a low level of usage or effective community management of unscheduled care needs. Interestingly, only $25 \%$ of admitted patients who chose this form of help were actually seen by a GP, although the overall numbers are small and should be interpreted with caution. Awareness of the out-of-hours primary care service could be improved.

Of the 12 subsequently admitted patients who contacted the out-of-hours primary care service first, three (25\%) were initially referred to NHS Direct or a walk-in centre. A further 15 patients chose one of these as their initial point of contact and one patient visited a community pharmacist. The overall contribution of these newer unscheduled care services in patients who are eventually admitted to hospital thus appears to be small. It is, however, impossible to comment on their general 
Figure 2 Actions and outcomes for patients seeking help (all percentages refer to the whole sample of 200).

${ }^{*}$ All patients contacting NHS Direct, attending a walk-in centre or consulting a community pharmacist were subsequently referred to the emergency department (ED).

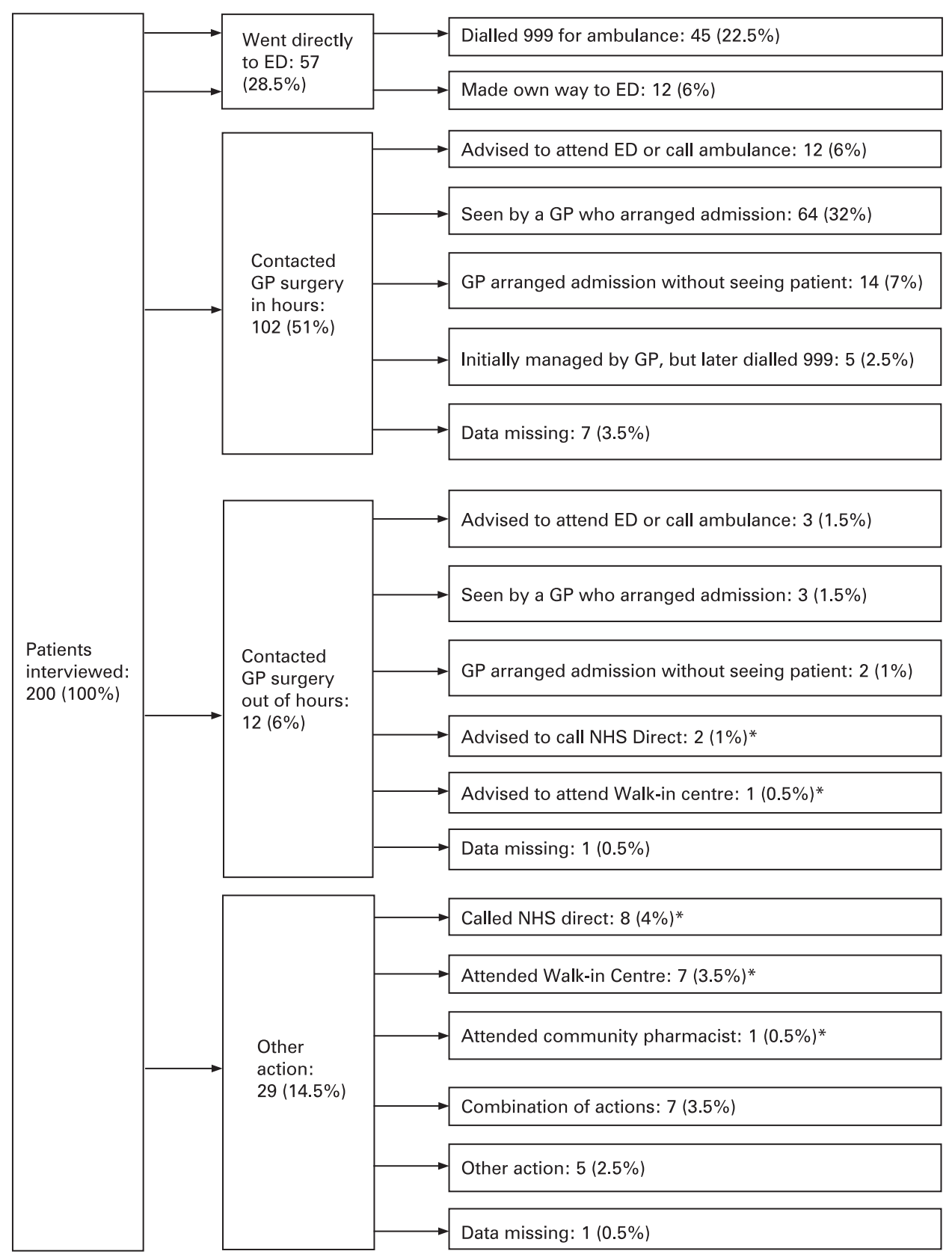

effectiveness as we only studied admitted patients and not the large numbers who use these facilities without subsequent hospital admission.

The view of our local healthcare commissioners was that universal primary care gate-keeping would reduce hospital admissions, although this is at odds with the patient choice agenda and the wider range of options now available. Only one

Table 2 Top five reasons why patients chose to contact their GP during normal office hours $(\mathrm{n}=102)$

\begin{tabular}{ll}
\hline Reason & No (\%) \\
\hline Problem not particularly urgent or severe & $35(34)$ \\
Calling the GP is the correct first step in accessing healthcare & $15(15)$ \\
Desire to avoid hospitals & $7(7)$ \\
Previous experience & $7(7)$ \\
Worsening of a chronic condition & $6(6)$ \\
\hline
\end{tabular}

quarter of admitted patients came directly to the ED, however, most commonly because they perceived their problem to be severe or urgent. It therefore appears that adopting measures to increase the number of patients contacting primary care first is unlikely to have a significant impact on hospital admissions,

Table 3 Top five reasons for not contacting the GP first $(\mathrm{n}=72$ )

\begin{tabular}{ll}
\hline Reason & No (\%) \\
\hline Perceived severity or urgency of their condition & $21(29)$ \\
Primary care services are not available out of hours & $16(22)$ \\
Contacting a GP will lead to unacceptable delays & $12(17)$ \\
Unable to attend the GP's surgery due to a lack of mobility & $8(11)$ \\
Contacting a GP will inevitably lead to ED referral & $4(6)$ \\
Not registered with a GP & $2(3)$ \\
\hline ED, emergency department. &
\end{tabular}

Patients or accompanying relatives were also asked what they would do if the same problem occurred in the future $(n=200)$. The results are shown in table 4 . 
Table 4 Intended actions if the same problem occurred again in the future $(\mathrm{n}=200)$

\begin{tabular}{ll}
\hline Reason & No (\%) \\
\hline Would call an ambulance or attend the ED directly & $69(35)$ \\
Contact the GP within normal working hours & $93(47)$ \\
Contact the out-of-hours primary care service & $7(4)$ \\
Call NHS Direct & $7(4)$ \\
Attend a walk-in centre & $5(3)$ \\
Attend a community pharmacist & $0(0)$ \\
Other, or a combination of actions & $19(10)$ \\
\hline
\end{tabular}

ED, emergency department.

particularly when the added factor of bystanders calling ambulances for persons taken ill in public, which would be very difficult to modify, is taken into account.

At the time that this study was conducted there had been recent substantial changes in UK general practice, with the responsibility for out-of-hours care passing from individual GPs to Primary Care Trusts and increasing delivery by GP cooperatives. ${ }^{8}$ This will have had an effect on the actions taken by patients and may alter over time as the population becomes more accustomed to the new system. Only a third of GPs now undertake work out of hours ${ }^{9}$ and chronic disease management forms an increasing part of the primary care workload. ${ }^{10}$ It has even been proposed that future GPs do not all need to be trained in out-of-hours work. ${ }^{11}$ At the same time, the specialty of emergency medicine is rapidly evolving, ${ }^{12}$ leading to a shift in key areas of unscheduled care expertise from primary care to ED. Other ED in the United Kingdom have recently recorded a steady increase in patients admitted via the ED, accompanied by a corresponding drop in those admitted directly to a ward. This is attributed to changes in the way that patients and GPs are using the service (R McGlone, personal communication).

There are a number of weaknesses in our study. The sample is relatively small and was taken from a single, inner-city teaching hospital. Inevitably we have only interviewed patients admitted to hospital and we have no data from the large number of patients managed entirely within primary care. Similarly, we did not set out to assess whether hospital admission was appropriate and are therefore unable to comment on this issue. Although our sample includes weekends and evenings it does not encompass the period between midnight and 08:00 hours, when patterns of activity may be different. The information offered by patients and their relatives may not have been wholly accurate, particularly concerning their stated reasons for taking certain actions. We sought to assure confidentiality, however, and encourage honest disclosure throughout the study. Only 10 patients declined to participate, but we did not interview 47 patients in triage category 1. Given the severity of their illness direct ED attendance by emergency ambulance is likely to be the preferred, and optimal, route of access for this patient group.

Our research could be usefully expanded to include more patients and centres over a longer time period and $24 \mathrm{~h}$ a day. It would be particularly informative to interview all patients attending the ED and not just those admitted to hospital. We hope that this will prove possible in the future.

\section{CONCLUSION}

We found that the majority of adult patients who are admitted to hospital with an acute illness seek professional help from primary care in the first instance and only one quarter attend the ED directly. Those who did attend the ED generally perceived their problem as more urgent or severe, or had an ambulance called on their behalf when they became ill in a public place. Of those patients eventually admitted, 25\% who contacted their GP in hours, and at least $50 \%$ out of hours, were admitted to hospital without actually seeing a primary healthcare professional. Few admitted patients in this sample chose other routes of initial healthcare access, such as NHS Direct or walk-in centres.

The shift towards ED care appears to be partly driven by changes in general practice and unfamiliarity with the new arrangements for out-of-hours primary care. Measures to channel unscheduled health needs through primary care may not substantially reduce hospital admissions.

Acknowledgements: The authors are grateful to all the patients and relatives who agreed to take part in this study and to the staff of the ED, Medical Assessment Unit and Surgical Assessment Unit at the Bristol Royal Infirmary for their assistance in recruitment. Their thanks also go to Linda Prosser and Xanthe Whittaker for their help in developing the research protocol and to Rosemary Greenwood for statistical analysis and advice.

Funding: This study was funded by the United Bristol Healthcare Trust.

Competing interests: Both authors are employed by the United Bristol Healthcare Trust, which is an NHS acute trust providing hospital-based emergency care.

Ethics approval: The study was approved by the Central and South Bristol Research Ethics Committee.

\section{REFERENCES}

1. UK Department of Health. http://www.performance.doh.gov.uk/hospitalactivity/ data requests/a and e attendances.htm (accessed 2 Feb 2006).

2. UK Department of Health. Available at: http://www.performance.doh.gov.uk/ hospitalactivity/data requests/admissions_from_ae.htm (accessed 2 Feb 2006).

3. Shepperd $\mathbf{S}$, lliffe $\overline{\mathrm{S}}$. Hospital at home versus in-patient hospital care. Cochrane Database Syst Rev 2001;(3):CD000356. doi: 101002/14651858. CD000356. pub 2.

4. Santos-Eggimann B. Increasing use of the emergency department in a Swiss hospital: observational study based on measures of the severity of cases. BMJ 2002;324:1186-7.

5. Edwards M. Primary care modernisation and A\&E attendances (letter). UK Department of Health: Gateway Reference 3531, 15 July 2004. http://www.dh.gov. uk/assetRoot/04/08/59/29/04085929.pdf (accessed 2 Feb 2006).

6. Department of Health. Our health, our care, our say: a new direction for community services. London: HMSO, 2006.

7. Lloyd G, Benger JR, Kaye P, et al. National service framework fails to address the decision time. Emerg Med J 2003;20:208.

8. Murie J. Doc around the clock. BMJ Careers (GP Ed) 2005;3 December:237-9.

9. Curtis M. Is the grass greener over in GP land? Hospital Doctor 2006;12 January:1823.

10. Cole A. UK GP activity exceeds expectations. BMJ 2005;331:536.

11. Chapman R. Training for out of hours work-core, or optional addition? BMJ Careers 2005;8 October:161-2.

12. Hughes G. Dizzy times for our specialty. Emerg Med J 2006;23:166. 